\title{
Drum rhythm spaces: from global models to style-specifc maps
}

\author{
Daniel Gómez-Marín ${ }^{1}$, Sergi Jordà ${ }^{1}$, and Perfecto Herrera ${ }^{1}$ \\ Universitat Pompeu Fabra - Music Technology Group \\ daniel.gomez@upf.edu
}

\begin{abstract}
This paper presents two experiments carried out to find rhythm descriptors that allow the organization of drum patterns in spaces resembling subjects similarity sensations. We revisit rhythm spaces published by Alf Gabrielsson in 1973, based on subject similarity ratings of drum rhythms from an early drum machine, and construct a new rhythm space based on similarity judgments using contemporary electronic dance music (EDM) patterns. We observe how a specific set of descriptors can be used to reconstruct both Gabrielsson's and the new EDM space, suggesting the descriptors capture drum similarity sensations in very different contexts. The set of descriptors and the methods employed are explained with detail and the possibility of having method for organizing rhythm patterns automatically is discussed.
\end{abstract}

Keywords: Rhythm Space, Electronic Dance Music (EDM), Drum Patterns, Rhythm Representations, Music Cognition, Conceptual Maps.

\section{Introduction}

Today there is an abundance of digital resources for Electronic Dance Music (EDM) composition: Gigabytes of one-shot drum sample libraries, drum loops and any other building block in the EDM production pipeline can be easily acquired and even freely downloaded from the internet. Although this proliferation can be very positive, it has an adverse effect which happens at the actual moment of starting to work and browsing (perhaps alphabetically or by date) through a collection of hundreds of poorly labeled musical files. Abundance in digital music production, and in any other context where work is based on digital media, becomes a problem. Therefore useful tools for dealing with specific information in an ordered and user centered manner must be developed. In this paper we focus on how to take advantage of abstract mappings (i.e., perceptual spaces, motivated by the specificities of human rhythm perception) to improve over the experience of organizing and retrieving audio or symbolic drum patterns.

For humans, processing and understanding a collection of items, as drum patterns, is accomplished by creating relations among those items and with other manifestations of them that have been previously experienced [5]. In this process the notion of similarity is indispensable for dealing with perceived phenomena and linking them to past experiences in an organized manner. In our scenario, 
a notion of similarity must be developed in order to systematically define relations among drum patterns, so they can be connected forming a similarity-based structure. This structure can then be suited for developing knowledge about a collection, visualizing it and even interacting with what is contained in it. This has been exemplified in many domains as color [19], timbre [11] and pitch [14] representations. This structure composed of a set of drum patterns is what we define as rhythm space, a collection organized geometrically based on similarity.

Previous research on drum rhythm spaces and similarity has been carried out by different authors [3] [6] [7]. Gabrielsson [7] performed several studies with polyphonic drum patterns, and his experiments resulted in rhythm spaces obtained by applying multidimensional scaling (MDS) on subject similarity ratings of drum patterns. According to his results rhythm spaces spanned from 2 or 3 axes that were given a certain perceptual and musical meaning such as meter, pulse or syncopated accentuation, onset density and rhythm complexity. We consider this research as one of those that sewed models for polyphonic rhythm perception.

As Gabrielsson's interpretations of the axes spanning his spaces are educated guesses without strong grounding on empirical data, we approach them with the help of rhythm descriptors that capture essential aspects of rhythm patterns. By analyzing the descriptors and positions of the patterns in Gabrielsson's spaces with a Lasso regression we expect to find a set of descriptors that captures the essence of subjects ratings revealed through the structure of the spaces. We hypothesize that such a set of descriptors can be used in a new context to predict how subjects would rate the similarity between a collection of other style's drum patterns. As we said before, EDM provides a fertile ground to research on theoretical and applied aspects of rhythm representation. In order to test this idea we carry out two experiments, one to evaluate how accurately one set of descriptors predicts Gabrielsson's spaces, and a second one to evaluate how the same set of descriptors predicts the organization of EDM patterns in our new space. Our results show that a specific subset of descriptors can be used to predict human similarity ratings and construct the spaces derived from them. We discuss this relevant set of descriptors, and the possibility of using them in a general method for automatic creation of rhythm spaces.

\section{Background}

Rhythms are commonly described with the use of notions as syncopation and event density. Syncopation is based on the idea that different metric weights for different regions of a sequence [15][16] are mentally constructed once subjects listen to a it and entrain with pulse and metric sensations. When a drum hit, an instrument note in a pattern, is played in a position with low metrical weight and it precedes a silence in a high metrical position we experience a tension with the pulse which is called a syncopation. Evidence of this tension has been studied from a neurological perspective observing an activation of the brain as the syncopated onset challenges the hierarchies imposed by the pulse [4][17]. These 
findings imply the coding of a rhythmic pattern goes deeper than the notes and silences found on its surface, so a framework based on pulse entrainment is essential for a better comprehension of rhythm cognition. Syncopation has also been found to play a fundamental role in our assessment of rhythm similarity of monophonic patterns [13][2][8][9]. Most of these research on rhythm and syncopation is based on monophonic patterns patterns but, in order to create notions of rhythm similarity useful in musical scenarios, polyphony must be addressed.

Some recent research in polyphonic syncopation [21][12] seeks to explain the effect that simultaneous layers of rhythmic patterns with different instruments have on the perceived syncopation sensation. These papers suggest that the general syncopation sensation of a polyphonic drum pattern is related to hierarchies and also timbre. Specifically, the central frequency of each individual sound present in a polyphonic percussive sequence is relevant to define the overall syncopation sensation. Experiments show how the effect of a kick drum (a low-frequency insrument) is more salient than that of a hi-hat when measuring syncopation on a polyphonic drum pattern. In these experiments patterns are segmented in three distinct layers covering different parts of the audible spectrum, which proves fundamental to compute a polyphonic syncopation value that captures the syncopation judgements of human listeners. Analyzing a percussive pattern by grouping its acoustic information, in low, mid and high bands, seems intuitively related to human perception as a mechanism perhaps to provide distinction while avoiding frequencial overlapping between instruments.

In this research we have created straightforward descriptors combining the concepts of syncopation, density, instrumentation and adapting the three frequency layers presented above. Our descriptors differ from others used in rhythm classification approaches (i.e. [10]) as ours are i) based on notions of human rhythmic processing, and ii) not based on audio signal analysis. Taking advantage of the typical acoustics of percusion sounds we define a mapping from the General MIDI Level 1 Percussion Key Map ${ }^{1}$ to three insrument categories (low, mid and high) based on the spectral center of the sound (i.e. a low tom belongs to low frequency and a snare to the mid frequency instruments).

Table 1 presents the list of descriptors used in this research, where the concepts of syncopation, frequency range and density (the amount of onsets per time unit) are combined using basic algebraic formulas to define quantifiable measures that we presume can be useful when making sense of a group of patterns.

\section{Experiment 1: Determining relevant rhythm descriptors to build Gabrielsson's spaces}

The studies by Gabrielsson [7] on rhythm spaces bring together the theory of cognitive spaces [20] applied to rhythm perception. In experiments 1 and 2 sub-

\footnotetext{
${ }^{1}$ The General MIDI standard has a list of 46 percussive instruments which are mapped one-to-one to a specific note. This is used to indicate what sort of sound will be heard when that note number is selected on a General MIDI synthesizer. https://www.midi.org/specifications/item/gm-level-1-sound-set
} 
Table 1. Table 1 list of used symbolic descriptors. For the detailed algorithms please visit the code repository: https://github.com/danielgomezmarin/rhythmtoolbox

\begin{tabular}{|c|c|}
\hline Name & Description \\
\hline number of instruments (NOI) & The sum of different instruments used in a pattern. \\
\hline loD, midD, hiD & $\begin{array}{l}\text { Sum of onsets for each different instrument group, } \\
\text { divided by the total number of steps in the pattern. }\end{array}$ \\
\hline stepD & $\begin{array}{l}\text { Sum of the steps in the pattern which contain at least } \\
\text { one onset, divided by the total amount of steps. }\end{array}$ \\
\hline lowness, midness, hiness & $\begin{array}{l}\text { Share of the total density of patterns that belongs } \\
\text { to each of the different instrument categories. } \\
\text { Computed as the quotient between the densities } \\
\text { per instrument category and the total density. }\end{array}$ \\
\hline losync, midsync, hisync & $\begin{array}{l}\text { Syncopation value computed for each instrumental } \\
\text { group's monophonic pattern. }\end{array}$ \\
\hline losyness, midsyness, hisyness & $\begin{array}{l}\text { Quotient of the syncopation value and the sum of } \\
\text { onsets for each instrument group. }\end{array}$ \\
\hline Polysync & Polyphonic syncopation as proposed by Witek et al. [21] \\
\hline
\end{tabular}

jects are exposed to different patterns with constant tempo and asked to rate their similarity, thus the reported distances and therefore the resulting spaces express similarity sensations based solely on the rhythmic constitution of the patterns. We want to find which descriptors are able to reproduce the spaces resulting from Gabrielsson's experiments as they are related to the mechanisms his subjects used to compare between the drum patterns. Once we achieve this goal, we further discuss that such descriptors can be used to automate rhythm classification tasks for a different collection of patterns.

\subsection{Methods}

Materials The patterns used by Gabrielsson are factory presets of the Ace Tone Rhythm Ace FR-3 ${ }^{2}$ drum machine which were recorded to magnetic tape. The patterns are Foxtrot, Habanera, Rock'n'roll, Rhumba and Beguine in Gabrielsson's experiment 1 (E1) and the same patterns plus Waltz in experiment 2 (E2) (see Figure 2). These patterns are a diverse sample of rhythms from different cultures and musical styles. The positions of the patterns we use for our analysis are extracted from Gabrielsson's paper.

Procedure We transcribe each pattern to MIDI and extract all descriptors from them. Then we run a Multi Task Lasso regression (alpha 0.03) [18] using the

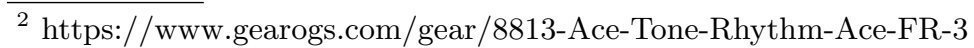




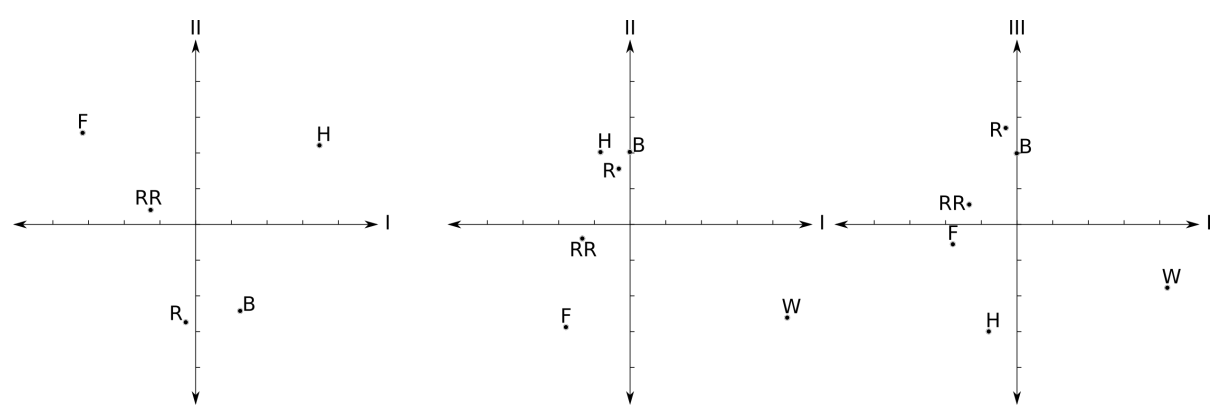

Fig. 1. Two spaces resulting from Gabrielsson's first two experiments. Space1(left) F:foxtrot, RR:rocknroll, R:rhumba, B:beguine, H:habanera. Space2 (center and right) $\mathrm{R}, \mathrm{B}, \mathrm{RR}, \mathrm{H}, \mathrm{F}$ and W:waltz. Gabrielson's paper is dedicated to interpreting the axes. Refer to the original paper for details.

positions of the patterns in each space as a target and the matrix of descriptors as variables. The Lasso regression returns a subset of descriptors and weights that maximizes correlation with the positions of the patterns in each space.

\subsection{Results}

The output of the Lasso analysis shows that using this set of descriptors (see table 1) [midD, hiD, hiness, lowsync, hisyness] the results of both axes of Gabrielsson's E1 are perfectly linearly correlated. For E1 and $\mathrm{x}$ axis the weights -0.660 , $-0.86,-0.068,-0.266,0.118$ respectively present a Spearman correlation of 0.999 (p-value $<0.005)$. For E1 and y axis the weights respectively present a Spearman correlation of 0.999 (p-value $<0.005$ ). For the space resulting from E2, Lasso analysis shows the descriptor set [midD, hiness, lowsync, midsync, hisync, losyness, hisyness] yields perfect correlations with its three axes. For E2 and $\mathrm{x}$ axis the weights $0.785,-0.073,0.242,0.574,-0.709,0.044,0.506$ respectively present a Spearman correlation of 0.999 (p-value $<0.005)$. For E2 and y axis the weights $0.333,-0.07,0.21,-0.032,1,0.002,-1.411$ respectively present a Spearman correlation of 0.942 and $\mathrm{p}$-value $=0.004$. For $\mathrm{E} 2$ and $\mathrm{z}$ axis the weights $0.313,-0.032$, $-1.12,0.104,0.81,-0.053,-0.724$ respectively present a spearman correlation of 0.999 (p-value $<0.005)$.

The sets of descriptors and weights for E1 and E2 perfectly describe the space reported by Gabrielsson and thus might be related with the overall polyphonic rhythm similarity sensations from which the spaces were created. It could be fair to presume that, if the spaces capture a similarity sensation, these descriptors play a role in our perception of polyphonic similarity that could go beyond its particular application in Gabrielsson's experiments 1 and 2. 

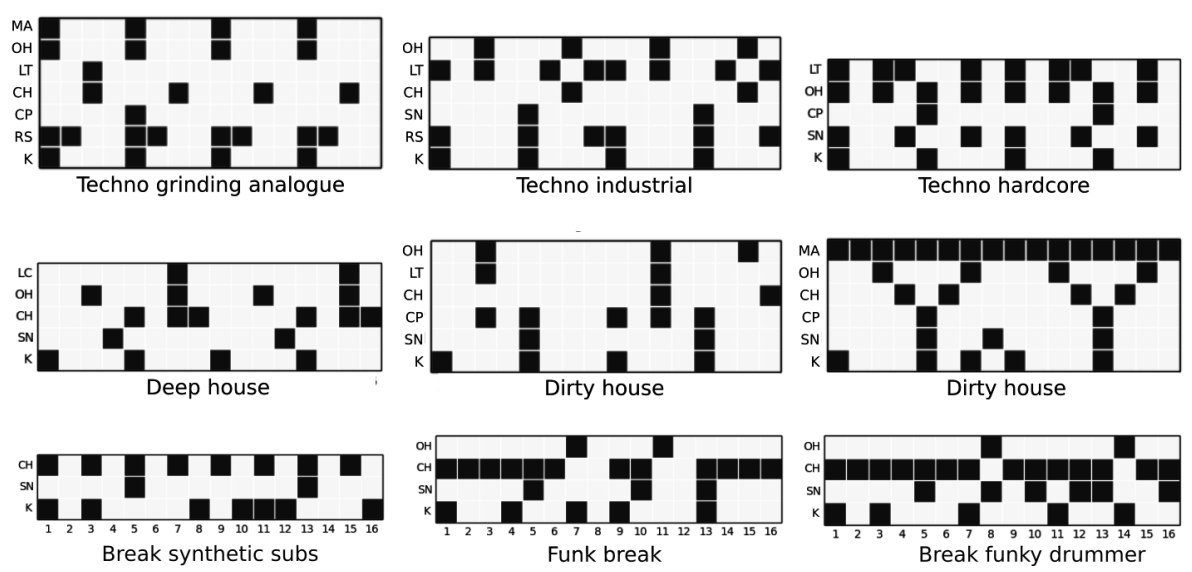

Fig. 2. Piano roll visualization of the nine EDM patterns. Steps on the $\mathrm{x}$ axis, onsets in dark.K: Kick Drum, S: Snare, CP: Clap, CH: Closed Hi-Hat, OH: Open Hi-Hat, LT: Low Tom, LC: Low Conga, MA: Shaker.

\section{Experiment 2: Generalization power of the features}

In the previous experiment we have found that small sets of descriptors can be used to describe Gabrielsson's E1 and E2 spaces. We then wonder how general these features are. In other words, are these features fitted to the particularities of the rhythms used by Gabrielsson or would they work when other, quite different, rhythm patterns are rated? From a computational point of view, we are proposing that, if we have a new set of drum patterns location on a humanbased rhythm space, we can extract only the descriptors defined on the previous experiment and by using a dimensional reduction technique as principal component analysis (PCA) or multidimensional scaling (MDS) we can predict with some accuracy the rhythm space. PCA finds a principal vector in the descriptor space in which the values of all descriptors are maximally dispersed and then additional orthogonal vectors are found to conform the predicted rhythm space. The other alternative, MDS, is based on a dissimilarity matrix of the patterns which is computed as the euclidean distance between the descriptor vectors of each pattern.

For this experiment we create an EDM rhythm space based on subject ratings following Gabrielsson's methodology: selecting a collection of EDM patterns and presenting pairwise combinations to subjects who report their similarity, and then, with those answers, creating a new rhythm space. An EDM drum rhythm collection is compiled specifically for this experiment. 


\subsection{Method}

Participants A total of 36 subjects participated in the survey, 5 females and 31 males, all had experience in music production and or musical training, according to a short survey they answered after finishing the listening task.

Materials In order to get a subject-based rhythm space, a set of rhythm patterns is needed. In order to select EDM-representative drum patterns, we turn to the EDM production literature printed and online and collect drum patterns explicitly associated to a certain EDM style. All patterns are 16 steps long, each step lasting for a 16th note. A total of 75 different patterns were collected, $70 \%$ of them belonged to the House (28\%), Breakbeat (26\%), and Techno (16\%) styles and the rest $30 \%$ belong to Garage, Drum n Bass, Hip-Hop, Trance, Chillout, Dubstep, Jungle and Trip-Hop styles. We create a preliminary rhythm space of the whole 75 pattern collection by extracting the complete list of aforementioned descriptors aforementioned and then using PCA. After dividing the preliminary space in nine equal-size rectangular areas we select one pattern from each one of them. In this way, the list of 75 patterns is reduced to 9 patterns (see table 2) intended to be representative enough of the variability of the included categories. The 9 patterns selected for the experiment are rendered to audio (in order to be played in the rating experiment) using single shot samples from the Roland 707, 808 and 909 drum machines. All selected patterns use instruments included in this set: Low Conga, Bass Drum, Side Stick, Maracas, Hand Clap, Snare, Closed Hi-Hat, Low Tom and Open Hi-Hat (Figure 2).

Table 2. Patterns selected from the sub-regions of the preliminary space. Left, center, right and top, center bottom represent the subdivisions of the space as explained in the text.

\begin{tabular}{cccc}
\hline & Left & Center & Right \\
\hline Top & Techno grinding analogue Techno industrial & Techno hardcore \\
Center & Deep house & Dirty house & Deep tech house \\
Bottom & Break synthetic subs & Funk break & Break funky drummer \\
\hline
\end{tabular}

Procedure A computer program is prepared to carry out the experiment. Before the subjects start the experiment, several patterns are presented to expose the timbre range of the percussive sounds used and also examples of identical, similar and completely different pairs of patterns. To evaluate all combinations between the 9 patterns, subjects rated the existing 36 possible pattern pairs in a triangular 9 element matrix (avoiding comparing a pattern with itself or repeating any pair). Additionally, 4 randomly selected pairs are presented twice 
for controlling the consistency of each subject's ratings, so, in total, subjects rate 40 pairs of patterns. The pairs are presented in a random order preventing any pattern to be in consecutive pairs. Before a pair is reproduced the order of its two patterns is also randomized so the same pair is presented in the two possible directions to different subjects. Subjects could listen to the same pair as many times as they needed and the similarity value was reported in a likert scale with a range from 1 to 10 where 1 means the pair is completely dissimilar and 10 means the pair had the topmost similarity (i.e., the pair contained equal patterns). When the subjects completed the experiment they answered some questions about themselves: age, gender, years of musical training, years of musical performance training, years of percussive musical performance training, hours per week spent attentively listening to music, experience in electronic music production, experience in electronic drum programming, number times listened to the pairs before answering. Finally, the possibility to leave a comment on the experiment is available.

\subsection{Results and discussion}

In order to simplify the analysis, the 10 point scale is mapped to a 5 point scale where each range of the new scale groups two values of the original scale (1 groups the results of 1 and 2, 2 groups 3 and 4 and so on). Three subjects rated different pairs as being exactly the same, and therefore they were discarded from the experiment because there were no identical pairs. The control pairs were used to perceive distortion in the ratings of the same pairs, and the average of the maximum difference of all subjects when rating the same pair is 1.8 units. In order to approximate our analysis to that of Gabrielsson we create a subgroup of subjects compliant with the musical background reported in his experiments, which is amateur musicians who had performed music for at least 4 years. A subset of our General Group composed of 18 subjects with at least 4 years of musical training was defined and we will refer to it as the Musicians group. For the General group, the inter quartile range (a measure of statistical dispersion) mean is 1.81 units and 1.48 units for the Musicians group suggesting more agreement in the Musicians sub-group. Pair $(1,3)$ presents a slight bimodal behaviour for the General Group which is reduced in the Musicians Sub group.

The observed means for each assessed pair present slight differences when both groups are compared. Only 9 pairs out of 36 differ in median value from one group to another: 6 pairs present changes in a degree of 2 units, and 3 pairs present changes in a degree of 1 . Pairs that involve rhythm Deep House do not change between groups and the pairs that involve rhythm 6 Deep Tech House have 4 changes between groups.

We can observe from the MDS that the three genres from where the rhythm patterns were extracted span across three distinct regions of the space (see Figure 3 ). Breakbeat patterns are located in the positive region of the $\mathrm{X}$ axis, while Techno and House patterns are located from the zero to the negative portion of the $\mathrm{X}$ axis. The $\mathrm{X}$-negative quadrants of the space contain in the $\mathrm{Y}$-positive region the Techno patterns and in the Y-negative region the House patterns. In 


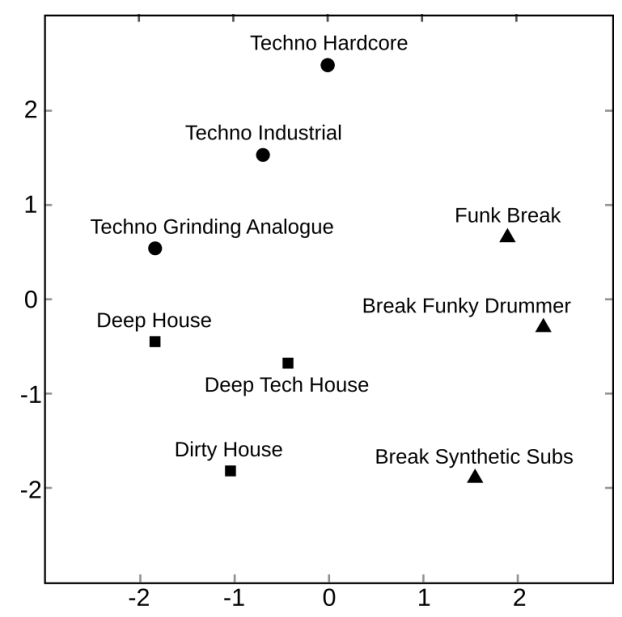

Fig. 3. Bi dimensional space obtained by using MDS on the dissimilarity matrix of subjects ratings.

EDM, rhythm and timbre are the most salient musical characteristics [1], so it is relevant for EDM drum patterns to retain stylistic/similarity information that comes through when subjects compare among them by listening.

Descriptor-based prediction of spaces We extract the two descriptor sets found in the previous experiment (by adjusting E1 and E2) from the patterns in the EDM space. Using those descriptor values we compute PCA and MDS to evaluate if the locations of the EDM patterns can be predicted with any of the sets. Table 3 presents the correlations between the predicted space and the resulting EDM space.

Table 3. Spearman rank correlations between each EDM axis and the forecasts using the descriptor sets from E1 and E2 with PCA and MDS.

\begin{tabular}{ccc}
\hline & $\mathrm{X}$ & $\mathrm{Y}$ \\
\hline E1 set PCA & $\rho=0.62 \mathrm{p}$-value $=0.076$ & $\rho=0.68 \mathrm{p}$-value $=0.042$ \\
E1 set MDS & $\rho=0.67 \mathrm{p}$-value $=0.049$ & $\rho=-0.78 \mathrm{p}$-value $=0.012$ \\
E2 set PCA & $\rho=0.52 \mathrm{p}$-value $=0.154$ & $\rho=0.45 \mathrm{p}$-value $=0.224$ \\
E2 set MDS & $\rho=0.63$ p-value $=0.067$ & $\rho=0.683 \mathrm{p}$-value $=0.042$ \\
E1+E2 set PCA $\rho=0.466$ p-value $=0.205$ & $\rho=0.683 \mathrm{p}$-value $=0.042$ \\
E1+E2 set MDS $\rho=0.383$ p-value $=0.308$ & $\rho=0.5 \mathrm{p}$-value $=0.17$ \\
\hline
\end{tabular}


Using the set of descriptors E1 for analyzing the patterns of the EDM experiment and then applying MDS to those results (E1 set MDS), we observe correlations of $=0.67(\mathrm{p}$-value $<0.05)$ and $=-0.78(\mathrm{p}$-value $<0.05)$ for $\mathrm{x}$ and $\mathrm{y}$ axis respectively. The other two combinations of sets and dimensional reduction techniques that are borderline correlated with the EDM space are, E1 set PCA and E2 set MDS but none of them have statistical significant correlations for any of the axes. The descriipttor set generated from E2 is not a good predictor of the patterns of the EDM space. This could be explained because in E2, one of the used patterns was ternary, hence probably introducing some "distortion", whereas in ours, all of the patterns are binary. The method of using the E1 set and then MDS captures the distance sensations reported by the subjects both in Gabrielsson's experiment 1 and and also in our EDM experiment.

From EDM to E1 and E2 The inverse process, making a Multi-Output Lasso analysis to extract a set of EDM well-correlated descriptors and then finding how they can predict the E1 and E2 spaces was also explored. In this case, the resulting descriptor set that perfectly correlates ( $p$-value $<0.05)$ with the EDM axes was different. However the predictions towards the E1 and E2 spaces, either by using PCA or MDS with the EDM set, were not statistically significant. This is expected as Gabrielsson's patterns are much different among them, representing six different musical styles, and thus cover a large perceptual/musical space, while the patterns used in the EDM experiment are variations of three styles which cover a small-scale rhythm space. In this case the macro scale descriptors can predict the small-scale space but not the opposite.

\subsection{Relevant set of descriptors}

The descriptors derived from the Multitask Lasso analysis that has the best fit with Gabrielson's E1 experiment and our EDM experiment using MDS are [midD, hiD, hiness, lowsync, hisyness]. These descriptors cover all frequency ranges in which the drum patterns are segmented (low, mid and high). The only low frequency descriptor present is lowsync which is expected given the crucial importance of the syncopation of the low frequencies in the overall syncopation sensation of a drum pattern, as proposed by Hove et al.[12]. The mid frequency range descriptor midD represents the normalized onset density of the mid frequency. The high frequency descriptors are hiD, hiness and hisyness, all related with teh density and the syncopation of the instruments mapped to the high frequency category (see table 1 for details on each descriptor).

\section{General Discussion}

We have discovered descriptors that allow to construct very general rhythm spaces that were reported long ago but withouth such descriptors-based analysis. Then we have seen that those descriptors, based on main concepts of rhythm cognition, allow to construct spaces constrained to EDM which even have a 
stylistic significance. Consequently, we could use those descriptors in systems that present, visualize and manipulate pattern collections, so that users have 2D representations close to their mental representations which could be exploited for search, selection and invention tasks.

Our results show how using a reduced set of descriptors, then computing Euclidean distance between the descriptor vectors and then using MDS, a subjectbased space composed of EDM patterns is reproduced with significant correlation values. Although this is a step forward towards a system capable of automatic drum pattern organization, further experiments must be carried out to evaluate its robustness in larger datasets.

Automatically visualizing in $2 \mathrm{D}$ a large amount of drum patterns has powerful implications in music production. The fact that a collection can be graphically explored and retrieved in a map in which similar patterns close whereas they appear far from different ones can improve the experience of music production and performance. On top of this map, we are currently developing drum pattern cross-fading algorithms adding a generative functionality to rhythm spaces. New patterns, not present in the collection, can emerge, therefore converting a discrete discrete rhythm space into a continuous one, and expanding an original "mimetic" space into a space for discovery.

Although the reported experiments were not designed for classification, it is revealing that the concept of EDM style comes through in the space resulting from our second experiment. It can be seen as a demonstration of how musical concepts as House, Techno and Breakbeat emerge based on listening to a handful of instances, each occupying a specific region of a conceptual space. As $61 \%$ of the Musicians group reported having experience in EDM production, the distribution by styles can also represent the effect of a previous context in EDM knowledge affecting how patterns are perceived and their similarity judged.

\section{Acknowledgments}

This research has been partially supported by the EU funded GiantSteps project ${ }^{3}$ (FP7-ICT-2013-10 Grant agreement nr 610591).

\section{References}

1. Butler, M.J.:Unlocking the groove: Rhythm, meter, and musical design in electronic dance music. Indiana University Press, (2006).

2. Cao, E., Lotstein, M., Johnson-Laird, P. N.:similarity and families of musical rhythms. Music Perception: An Interdisciplinary Journal, 31(5), 444469 (2014).

3. Esparza, Tlacael Miguel, Juan Pablo Bello, and Eric J. Humphrey. "From genre classification to rhythm similarity: Computational and musicological insights." Journal of New Music Research 44.1 (2015): 39-57.

\footnotetext{
$\overline{{ }^{3} \text { http://www.giantsteps-project.eu }}$
} 
4. Fujii, S., Schlaug, G.: The Harvard Beat Assessment Test (H-BAT): a battery for assessing beat perception and production and their dissociation. Frontiers in human neuroscience, 7, 771 (2013).

5. Gärdenfors, P.: Conceptual Spaces: The Geometry of Thought. Book (1st ed.). The MIT Press, Cambridge, Massachussetts (2000).

6. Gabrielsson, A.: Similarity ratings and dimension analyses of auditory rhythm patterns: I. Scandinavian Journal of Psychology, 14, 138160 (1973).

7. Gabrielsson, A.: Similarity ratings and dimension analyses of auditory rhythm patterns: II. Scandinavian Journal of Psychology, 14(3), 161176 (1973).

8. Gómez-Marín, D., Jordà, S., Herrera, P.: Strictly Rhythm: Exploring the effects of identical regions and meter induction in rhythmic similarity perception. In 11th International Symposium on Computer Music Multidisciplinary Research (CMMR), Plymouth (2015).

9. Gómez-Marín, D., Jordà, S., Herrera, P.: Pad and Sad: Two Awareness-Weighted Rhythmic Similarity Distances. In 16th International Society for Music Information Retrieval Conference ISMIR, Málaga (2015).

10. Gouyon, Fabien, et al. "Evaluating rhythmic descriptors for musical genre classification." Proceedings of the AES 25th International Conference. 2004.

11. Grey, J. M.: Multidimensional perceptual scaling of musical timbres. The Journal of the Acoustical Society of America, 61(5), 12701277 (1977).

12. Hove, M. J., Marie, C., Bruce, I. C.,Trainor, L. J.: Superior time perception for lower musical pitch explains why bass-ranged instruments lay down musical rhythms. Proceedings of the National Academy of Sciences, 111(28), 1038310388 (2014).

13. Johnson-Laird, P. N.: Rhythm and meter: A theory at the computational level. Psychomusicology: A Journal of Research in Music Cognition, 10(2), 88106 (1991).

14. Krumhansl, C. L.: The psychological representation of musical pitch in a tonal context. Cognitive Psychology, 11(3), 346374 (1979).

15. Longuet-Higgins, H. C., Lee, C. S.: The Rhythmic Interpretation of Monophonic Music. Music Perception: An Interdisciplinary Journal, 1(4), 424441 (1984).

16. Palmer, C., Krumhansl, C. L.: Mental representations for musical meter. Journal of Experimental Psychology. Human Perception and Performance, 16(4), 728741 (1990).

17. Patel, A.D., Iversen, J.R.: The evolutionary neuroscience of musical beat perception: the Action Simulation for Auditory Prediction (ASAP) hypothesis. Frontiers in Systems Neuroscience, 8, p.57 (2014).

18. Pedregosa, F., Varoquaux, G., Gramfort, A., Vincent, M., Thirion, B., Grisel, O., Blondel, M.: Scikit-learn: Machine learning in Python. Journal of Machine Learning research (Vol. 12) (2011).

19. Shepard, R. N.: The analysis of proximities: Multidimensional scaling with an unknown distance function. II. Psychometrika, 27(3), 219246 (1962).

20. Shoben, E. J., Ross, B. H.: 7. Structure and Process in Cognitive Psychology Using Multidimensional Scaling and Related Techniques. In R. R. Ronning, J. A. Glover, J. C. Conoley, \& J. C. Witt (Eds.), The Influence of Cognitive Psychology on Testing. Hillsdale, NJ: Lawrence Erlbaum Associates (1987).

21. Witek, M. A. G., Clarke, E. F., Wallentin, M., Kringelbach, M. L., Vuust, P.: Syncopation, body-movement and pleasure in groove music. PLoS ONE, 9(4) (2014). 